\title{
Multi-Functional Consequences of a Single Mutation of CFTR of the Importance to Analyze Mutations by Functional Tests \\ Ladeveze $\mathbf{V}^{1^{*}}$, Farhat $\mathbf{R}^{1}$, El Seedy $\mathbf{A}^{1,2}$ and Kitzis $\mathbf{A}^{1,3}$ \\ ${ }^{1} E A 3808$, Université de Poitiers, Pôle Biologie Santé, France
}

${ }^{2}$ Department of Genetics, University of Alexandria, Aflaton St. 21545 El Shatby, Alexandria, Egypt

${ }^{3} \mathrm{CHU}$ de Poitiers, France

*Corresponding author: Veronique Ladeveze, EA3808, Université de Poitiers, Pole Biologie Sante, 1 rue G Bonnet, TSA51106, 86073 Poitiers cedex 9 , France, Tel: (33) 5494549 77; E-mail: Veronique.ladeveze@univ-poitiers.fr

Rec date: May 04, 2016; Acc date: Jun 13, 2016; Pub date: Jun 15, 2016

Copyright: (c) 2016 Ladevezef V, et al. This is an open-access article distributed under the terms of the Creative Commons Attribution License, which permits unrestricted use, distribution, and reproduction in any medium, provided the original author and source are credited.

\section{Abstract}

Cystic Fibrosis is the most frequent rare disease in the Caucasian population. This severe hereditary recessive disease is caused by mutations in the Cystic Fibrosis transmembrane conductance regulator (CFTR) gene. This gene encodes for a protein expressed at the apical membrane of epithelial cells. Mutations are classified in six classes based on their effects and phenotype severity. The F508delmutation is the most frequent mutation of the CFTR gene; it induces a misfolding of the protein thus blocking its maturation, membrane localization and in the end, its functionality.
\end{abstract}

This study combines a clinical approach and multiple level cellular analyses to determine the physiopathological consequences of the c.1392G>T (p.Lys464Asn) CFTR exon 10 mutation, detected in a CF patient with a frame shift deletion in trans and a TG(11)T(5) in cis. First, the splicing was studied by minigene experiments, with different $\mathrm{TG}(\mathrm{m}) \mathrm{T}(\mathrm{n})$ alleles, and by nasal cell mRNA extracts to determine the consequence of $c .1392 \mathrm{G}>\mathrm{T}$. Then, the processing of p.Lys464Asn protein was evaluated, in cellulo, by western blotting analyses.

The c.1392G $>\mathrm{T}$ mutation affects exon 10 splicing by inducing its complete deletion and encoding a frame shift transcript. The polymorphism TG (11)T(5) increases the effects of this mutation on aberrant splicing, suggesting the importance of complex allele. Analysis of mRNA obtained from maternal airway epithelial cells confirmed these in cellulo results. At the protein level the p.Lys464Asn protein showed no fully glycosylated form.

Thus, the c.1392G>T mutation alone or in association with the poly $\mathrm{T}$ tract revealed obvious impacts on splicing and CFTR protein processing. The c.[T(5); 1392G>T] complex allele contributes to the CF phenotype by affecting splicing and inducing a severe misprocessing defect. These results demonstrate that the classical CFTR mutations classification is not sufficient: in vivo and in cellulo studies of a possible complex allele in a patient are required to provide correct CFTR mutation classification, adequate medical counseling, and adapted therapeutic strategies.

Keywords: CFTR mutation; Cystic fibrosis; Complex allele; Clinical genetics; Cystic fibrosis; Molecular genetics; Splicing; Protein processing; Genotype phenotype correlation; Genetic counselling

\section{Introduction}

Cystic fibrosis (CF) is one of the most frequent autosomal recessive diseases in the Caucasian population and is due to CF transmembrane conductance regulator (CFTR) dysfunction. Milder forms, in which there is evidence of CFTR dysfunction but the diagnostic criteria for $\mathrm{CF}$ are not met, are termed CFTR-related disorders (CFTR-RD) (cystic fibrosis mutation database-CFMD, http://www.genet.sickkids.on.ca/ cftr/) [1]. Phenotype heterogeneity is partly due to CFTR mutational and genotype heterogeneity, interplaying with other genetic factors/ modifiers and environmental factors. Generally, patients with the classic form of CF have CF-causing mutations in each copy of the CFTR gene, whereas patients with a CFTR-RD may have a CF mutation in one copy and a mild mutation in the other allele, or mild mutations in both copies.
The CFTR gene (CFTR; NM_000492.3; MIM\# 602421) is located on the short arm of chromosome 7 at position $7 \mathrm{p} 31$. This $250 \mathrm{~kb}$ gene, containing 27 exons, appears highly susceptible to mutations due to its large size. Understanding how each mutation affects CFTR function as is crucial in informing the clinical approach and therapeutic strategies. Therefore, mutations are classified into one, or more, of the six established classes, according to their characteristics [2,3]. CFTR is involved in the active transport of ions through the apical membrane of epithelial cells [3]. So the CFTR mutations affect the quantity or function of CFTR channels. Class I is due to deletion of the gene or premature Stop codon or alteration of critical RNA signal resulting in failure to synthesize full-length CFTR. Class II induces little to no functional CFTR by improper folding resulting in defective maturation and trafficking. Two other classes present CFTR at the cell surface but one cause a defect in regulation that impairs opening of the CFTR chloride channel (Class III) and the other causes a structural defect in the CFTR channel that reduces the conductance of CFTR channel (Class IV). Mutations inducing RNA splicing lead to reduced quantity of functional CFTR (Class V). Mutations increasing cell surface turnover and degradation of CFTR belong to Class VI. 
Moreover, genotype-phenotype correlations in CF may be difficult to establish because of phenotype variability, which is associated with certain CFTR gene mutations and the existence of polymutant variants on the same allele, termed complex alleles. The effect of a mutation can vary when it is present in cis with another variant, modifying phenotype severity and thus complicating diagnosis and genetic counseling. For example, a complex allele containing two mild mutations can dramatically alter CFTR function [4,5] (Figure 1).

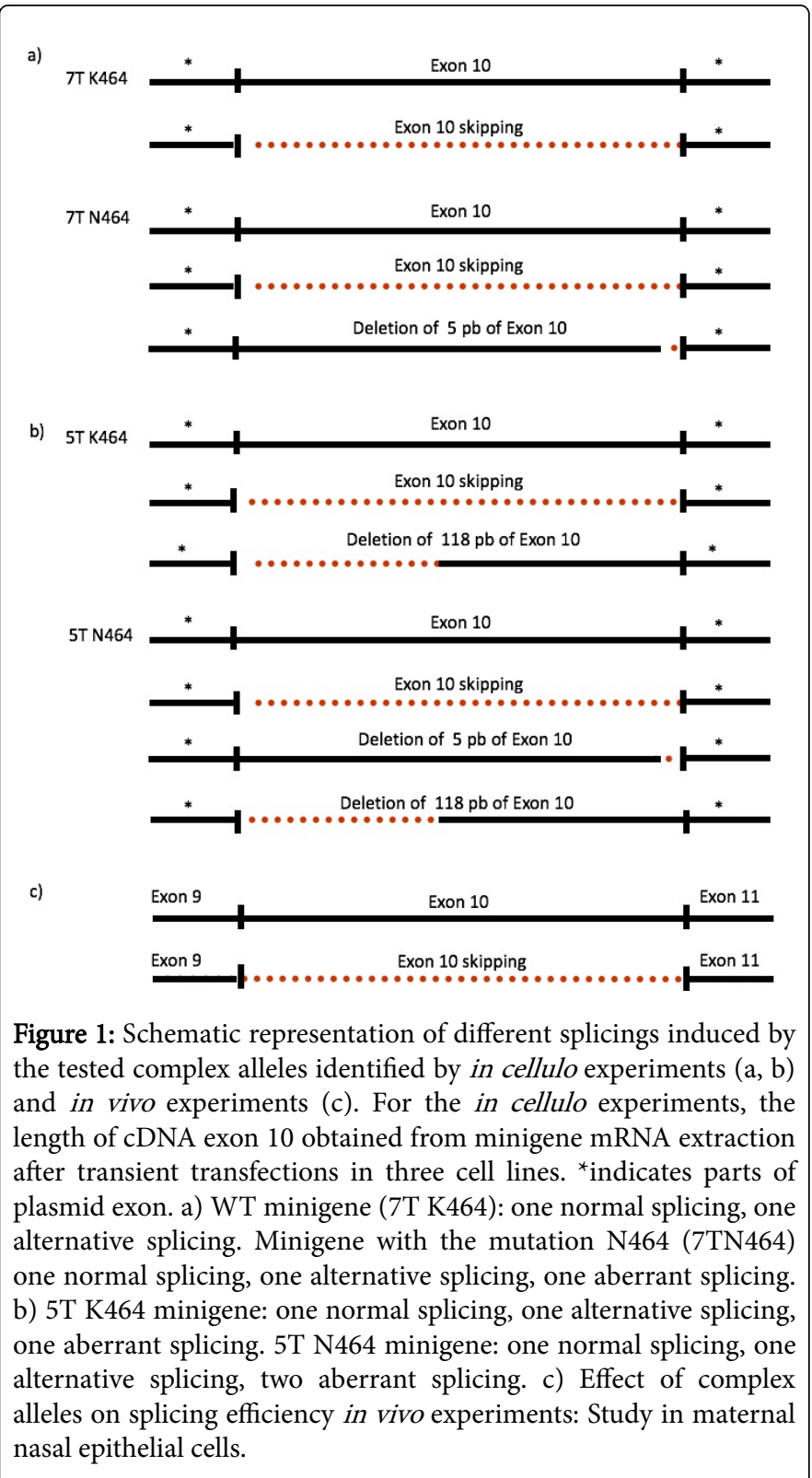

Previous studies on the CFTR gene have reported that exon 10 (new nomenclature according to the recommendations of the Human Genome Variation Society guidelines http://www.hgvs.org/ mutnomen/) and its flanking regions are present in multiple copies in the human genome [6,7]. These duplications present mutations compared to the normal exon 10 sequence. It could be difficult to determine if these mutations are present on CFTR exon 10 or on an ectopic exon 10 sequence. In a previous paper [8] we presented new
PCR conditions that allow specific amplification of CFTR exon 10 and its flanking regions and not the ectopic regions. By this methodology, we analyzed mutations described in the CF data base as being CFTR mutations but also found in ectopic regions [9].

Following this work our attention was particularly focus on one of this mutation: c.1392G $>\mathrm{T}$ variant (p.Lys464Asn). So, this mutation and/or complex allele carrying this mutation were studied to better understand its impact on pathophysiologic defects.

\section{Materials and Methods}

\section{Case history and family}

The c.1392G > T variant (p.Lys464Asn) was first identified in a CF child carrying a frameshift mutation in trans (c.3659delC) and manifesting severe CF clinical symptoms. In the studied CF patient, the sequencing of exon 10 and its flanking regions revealed that the $\mathrm{c}$. $1392 \mathrm{G}>\mathrm{T}$ mutation and the TG(11)T(5) polymorphism are associated in cis. Mutational analysis for the CFTR gene was carried out after written informed consent from the patients and under research protocols according to the declaration of Helsinki.

\section{Generation of minigene constructs for the in cellulo splicing analyses}

The pTBNdeI plasmid (generously provided by Professor Pagani) is a vector designed to analyze mutations suspected of being involved in AS in transfected mammalian cells. The construction and validation of the hybrid minigene used in this study has been described elsewhere [10]. The four inserts used in this study are: Insert 1 (c.[TG(11)T(7); 1392G]) and 2 (c.[TG(11)T(5); 1392G]) are obtained from patients and were inserted in the pTBNdeI plasmid. Plasmids containing inserts 3 (c.[TG(11)T(7); c.1392G>T]) and 4 (c.[TG(11)T(5); c. $1392 \mathrm{G}>\mathrm{T}]$ ) were obtained by directed mutagenesis realized on the plasmids. All hybrid minigene constructs were sequenced to verify the correct insertion of WT and mutated DNA fragments [11].

\section{Construction of CFTR-mutated cDNA for the in cellulo protein processing analyses}

The pTCF plasmid (generously provided by Dr. Fanen) is a vector designed to produce CFTR. In this study WT, c.1521_1523delCTT (p.Phe508del), and c.1392G $>$ T pTCF plasmids were used. The c. $1392 \mathrm{G}>\mathrm{T}$ plasmid was obtained by directed mutagenesis of the WT using the gene tailor site-directed mutagenesis kit (Invitrogen) and specific primers described previously [11]. The presence of the desired mutation was verified by sequencing.

\section{Cell culture and transient transfections}

Cell lines were grown in DMEM medium with Glutamax-I (Life Technologies) supplemented with $10 \%$ foetal bovine serum (Gibco), 100 units $/ \mathrm{mL}$ penicillin, and $100 \mu \mathrm{g} / \mathrm{mL}$ of streptomycin in a humidified incubator at $37^{\circ} \mathrm{C}$ in the presence of $5 \% \mathrm{CO}_{2}$. Cells were transiently transfected by WT and mutant CFTR plasmids using Lipofectamine 2000 (Invitrogen) according to the manufacturer's instructions. Transfections were performed in triplicate for western blotting (WB), and RNA extraction experiments. All three cell lines (HeLa, HT29 and HEK293) were used in the minigene experiments; for analyses, only HEK293 cells were used. 


\section{RT-PCR technique}

Total RNA was extracted from cell lysates using the RNeasy Mini Kit (Qiagen, Germany). cDNA synthesis was carried out at $37^{\circ} \mathrm{C}$ for 1 hour. The reaction was stopped by 2 minute incubation at $100^{\circ} \mathrm{C}$. RTPCR was performed using primers designed for the exons of plasmid or of CFTR (Table 1). PCR was performed using a 9700 GeneAmp Thermo Cycler (Perkin Elmer) under the cycling conditions described by Farhat et al. [11].

\begin{tabular}{|l|l|l|}
\hline \multirow{2}{*}{ G3/F2 } & $\begin{array}{l}\text { Sequencing cDNA of } \\
\text { cultured cells }\end{array}$ & 5'CAACTTCAAGCTCCTAAGCCACTGC3' \\
\cline { 3 - 3 } & $\begin{array}{l}\text { 5'AGGGTCACCAGGAAGTTGGTTAAATC } \\
\text { A3' }\end{array}$ \\
\hline \multirow{2}{*}{ Ex9/Ex11 } & $\begin{array}{l}\text { Sequencing cDNA of } \\
\text { epithelial cells }\end{array}$ & 5'GATGACGCTTCTGTATCTATAT3' \\
\cline { 3 - 3 } & 5'CTAATGGTGATGACAGCCTC3' \\
\hline
\end{tabular}

Table 1: Primers used in RT-PCR experiments.

\section{In cellulo evaluation of protein processing using WB analysis}

Twenty-four hours after transfection, cells were harvested and resuspended in RIPA (50 mM Tris- $\mathrm{HCl}$ (pH 7.5), 1 mM EDTA, and $100 \mathrm{mM} \mathrm{NaCl}, 1 \%$ Triton X-100) buffer supplemented with protease inhibitors (Roche Diagnostics). Cell lysates were incubated on ice for $30 \mathrm{~min}$ and clarified by centrifugation at $15,000 \mathrm{~g}$ for $10 \mathrm{~min}$ at $4^{\circ} \mathrm{C}$. Total proteins were quantified using the BCA protein assay reagent (Sigma), and $50 \mu \mathrm{g}$ of protein was analyzed on an SDSPAGE gel according to Laëmmli and Favre. Whole cell lysates were separated on a 5\% SDS-PAGE gel (BioRad Laboratories Inc.) and transferred onto nitrocellulose membranes. Membranes were first incubated with 5\% BSA in TBST (TBS; $0.05 \%$ Tween 20) for $2 \mathrm{~h}$ at $4^{\circ} \mathrm{C}$. The membrane s were then washed 3 times for $5 \mathrm{~min}$ in TBST, followed by incubation with primary antibody against CFTR (clone M3A7; Chemicon) $(1 / 2000$ in $1 \mathrm{X}$ PBS) overnight. Mouse anti $\beta$-actin monoclonal antibody ( $1 / 10000$ in $5 \% \mathrm{w} / \mathrm{v}$ dry milk) was applied to detect $\beta$-actin. Membranes were then washed 3 times with TBST and incubated with secondary antibody conjugated to Alexa Fluor 555 (1:100 in PBS; Invitrogen) for $6 \mathrm{~h}$. After further washing in TBST the blots were scanned with a Typhoon imager (GE Healthcare), using an excitation laser (532 nm) and a $580 \mathrm{~nm}$ band-pass filter (580 BP 30).

\section{Results}

The c.1392G $>\mathrm{T}$ variant affects the last nucleotide of exon 10 and induces the p.Lys464Asn substitution that occurs in the nucleotide binding domain 1 (NBD1) of CFTR. Aberrant Splicing (AS) and (or) protein misprocessing are two mechanisms that can explain the $\mathrm{CF}$ phenotype. After exhaustive medical and channel activity investigations conducted on the patient, fundamental studies were performed at the mRNA and protein levels to determine the proper genetic processes causing this CF phenotype [11].

\section{Co-implication of c.1392G $>$ T mutation and TG(m)T(n) polymorphism in CFTR exon $10 \mathrm{AS}$ revealed by in cellulo analyses}

Four plasmids were constructed with TG(11), as it is the most frequent allele in the general population and it is the genotype of the CF patient. After transfections (3 independant transfections in each tested line), mRNAs were analysed by RT-PCR and directly sequenced.
The Table 1 shows the different fragments obtained and their amount approximately.

Firstly, a 432 bp fragment, corresponding to the expected normal full-length mRNA, was obtained in the 4 tested haplotypes. Secondly, the c. $[T(7) ; c .1392 \mathrm{G}]$ genotype (considered WT) induced few Alternative Splicing.

Thirdly, the c.[T(5); c.1392G] genotype induced more AS: exon 10 skipping and production of a previously undescribed mRNA lacking the first $118 \mathrm{bp}$ of exon 10 corresponding to an aberrant splicing.

Fourthly, the c. $[\mathrm{T}(7) ;$ c.1392G $>\mathrm{T}]$ genotype induced both exon 10 skipping and a frame shift deletion of five nucleotides, corresponding also to an aberrant splicing.

Finally, the c.[T(5); c.1392G $>\mathrm{T}]$ genotype induced an abnormal pattern: the major product corresponds to the exon 10 skipping and two abnormal mRNAs lacking the first $118 \mathrm{bp}$ (to T(5)) or the last $5 \mathrm{bp}$ (due to c.1392G $>$ T) of exon 10 .

These results are likely to contribute to the CF phenotype in our patient. Indeed, the haplotype detected in the CF child causes AS (intense increase of exon 10 skipping due to complex allele, minus $5 \mathrm{bp}$ in $3^{\prime}$ due to the mutation and minus 118 bp in 5 ' due to the polymorphism $\mathrm{T}(5)$ ). Only the transcript lacking the whole of exon 10 does not induce a frameshift.

\section{Validation of CFTR exon 10 AS by in vivo analyses}

Native airway epithelial cells were obtained from the parents by nasal brushing using vaginal cytology brush (protocol generously given by Dr. Girodon). Cell samples were conserved in RNA later preservation solution (Qiagen, Germany).

Parental studies revealed that the c.[1210-34TG[11];1210-12T[5]; $1392 \mathrm{G}>\mathrm{T}]$ is the maternal allele.

Only 2 mRNAs were detected in maternal sapmles: one obtained by normal splicing carrying the mutation, and the other one by AS (exon 10 skipping). The sequencing of maternal cDNA does not reveal the presence of mRNA minus $5 \mathrm{bp}$ or $118 \mathrm{bp}$ as identified in the in cellulo experiments. It is certainly due to nonsense-mediated mRNA decay (NMD), as these RNAs are not in frame with normal CFTR mRNA [12].

\section{Severe misprocessing of p.Lys464Asn CFTR revealed by in cellulo protein processing}

As p.Phe508del, this mutated protein was restricted to intracellular compartments, and only a narrow band of approximately $140 \mathrm{kDa}$ (band B) is detected by Western blot, which represented the core glycosylated protein located in endoplasmic reticulum (ER), confirming the presence of a processing defect and a retention in the ER compartment (Figure 2).

Under the experimental conditions, two bands have been shown by western blot of WT-CFTR: the band B and the second, with an approximate molecular mass of $170 \mathrm{kDa}$ (band C), and represented mature, fully glycosylated protein that had migrated through the Golgi complex to the cell membrane [13]. 

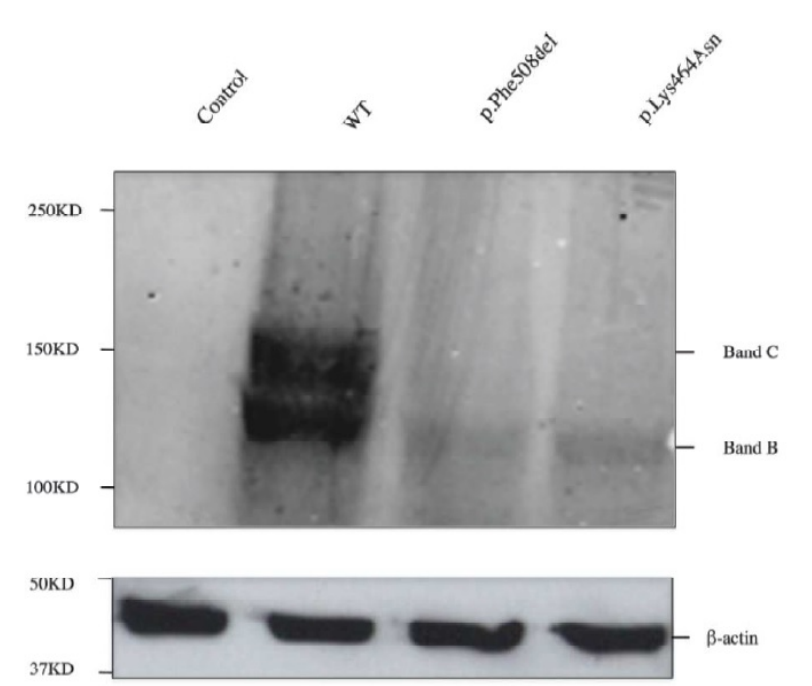

Figure 2: Processing of wild-type (WT) and mutant CFTR proteins studied by Western blotting. Wild-type (WT) and mutant CFTR (p.Phe508del and p.Lys464Asn) proteins were obtained from transiently transfected HEK293 cell line. CFTR protein was detected by M3A7 (Chemicon), a mouse monoclonal antibody that recognizes a C-ter epitote in the region of residues 1370-1380. Arrows on the right indicate the position of core-glycosylated (band B) and fully glycosylated (band C) forms of CFTR. Control corresponds to cells without transfection. The loading control is actin.

\section{Discussion}

The classification of c.1392G $>\mathrm{T}$ according to the established system of CFTR mutations classification is not straight forward.

c.1392G $>\mathrm{T}$ was associated with the increased production of a proportion of mRNA exhibiting exon 10 skipping. Thus, c.1392G $>\mathrm{T}$ can be considered a splicing mutation (Class V). The presence of this mutation in the 5' splice site (donor site) could affect the splicing by disturbing the binding of the U1 snRNA. Normally, to initiate splicing, this small nuclear RNA recognizes the donor site by complementary binding between its 5 ' end and a conserved sequence of the donor site [14]. The localization of c.1392G $>$ T mutation could affect this essential interaction for a normal exon definition.

In addition, the results showed that the complex allele c. [1210-12T[5];1392G $>\mathrm{T}]$ induces an intense increase of the rate of exon 10 skipping, suggesting Class I. The c.[1210-35TG[m];-12T[n]] polymorphism (or TG(m)T(n)) is located at 3' end of intron 9 and induces variable levels of exon 10 skipping depending on the number of TG repeats and the size of the poly-T tract [10-15]. The low number of $\mathrm{T}$ repeats could affect the splicing by reducing the distance separating the AG 3' intronic site from the UG repeats, here the TDP-43 protein binds [16]. In fact, this protein has been demonstrated to affect exon 10 splicing [17] and its affinity to mRNA is higher with a high number of UG [16]. Furthermore, the TDP-43 protein has a higher level of expression in a testicular cell line [16], which could explain the occurrence of CBVAD in $\mathrm{TG}(13) \mathrm{T}(5)$ patients carrying in trans a CF mutation.
Furthermore, the c.1392G $>$ T mutation also belongs to class II, as it prevents the maturation of the mutated protein and thus its translocation to the cell membrane. Conformational modifications caused by the p.Lys464Asn substitution may explain the observed ER retention, since the export of a protein from the ER to the Golgi is tightly coupled to the acquisition of a native conformation [18]. Therefore, the Lys464 amino acid may play an important role in threedimensional folding. The presence of an asparagine in this position may alter the protein, specifically the NBD1 domain, preventing formation of the proper conformation and resulting in ER retention, retrotranslocation, and ultimately cytoplasmic degradation of the mutated protein [18]. These results confirm that, even if the mutated mRNA is present, the resulting altered protein is unable to form a functional CFTR protein. Therefore, it could be considered that the CF phenotype results mainly from the misprocessing, since it has been demonstrated that only $4 \%$ of normally spliced non-mutated CFTR mRNA are sufficient to ensure an ordinary channel function [19].

In conclusion, the CF phenotype induced by $1392 \mathrm{G}>\mathrm{T}$ is due to both abnormal CFTR processing and splicing defects. In the particular case of the patient described in this study, the TG (11) T(5) polymorphism emphasizes the splicing defects, while in the case of a $\mathrm{TG}(11) \mathrm{T}(7)$ polymorphism the protein defect would be the principal origin of the $\mathrm{CF}$ phenotype.

Thus, this study illustrates the need for data from multiple sources to be taken consideration when CFTR mutations are classified. Moreover, the classification of a mutation is not sufficient to inform the clinical approach, as the presence of complex allele may alter the specific effect of a single mutation. Therefore, an individual approach to perform appropriate diagnosis and treatment should be provided where possible.

\section{Acknowledgments}

We thank Isabelle Sermet-Gaudelus for providing nasal epithelial cells. We also thank Catherine Adolphe for the technical assistance. This work was supported by "association mucoviscidose: ABCF2". Raed Farhat received a fello wship from CNRS-Lebanon and Dr. Ayman El-Seedy received a fellowship from the Egyptian Government. This work was supported by Poitiers Hospital and University of Poitiers, France and ABCF2 mucoviscidose, France.

\section{References}

1. Bombieri C, Claustres M, De Boeck K, Derichs N, Dodge J, et al. (2011) Recommendations for the classification of diseases as CFTR-related disorders. J Cyst Fibros 10 Suppl 2: S86-102.

2. Welsh MJ, Smith AE (1993) Molecular mechanisms of CFTR chloride channel dysfunction in cystic fibrosis. Cell 73: 1251-1254.

3. Zielenski J, Tsui LC (1995) Cystic fibrosis: genotypic and phenotypic variations. Annu Rev Genet 29: 777-807.

4. Clain J, Fritsch J, Lehmann-Che J, Bali M, Arous N, et al. (2001) Two mild cystic fibrosis-associated mutations result in severe cystic fibrosis when combined in cis and reveal a residue important for cystic fibrosis transmembrane conductance regulator processing and function. J Biol Chem 276: 9045-9049.

5. Clain J, Lehmann-Che J, Girodon E, Lipecka J, Edelman A, et al. (2005) A neutral variant involved in a complex CFTR allele contributes to a severe cystic fibrosis phenotype. Hum Genet 116: 454-460.

6. Rozmahel R, Heng HH, Duncan AM, Shi XM, Rommens JM, et al. (1997) Amplification of CFTR exon 9 sequences to multiple locations in the human genome. Genomics 45: 554-561. 
Citation: Ladeveze V, Farhat R, El Seedy A, Kitzis A (2016) Multi-Functional Consequences of a Single Mutation of CFTR of the Importance to Analyze Mutations by Functional Tests. Single Cell Biol 5: 142. doi:10.4172/2168-9431.1000142

Page 5 of 5

7. Liu X, Li X, Li M, Acimovic YJ, Li Z, et al. (2004) Characterization of the segmental duplication LCR7-20 in the human genome. Genomics 83: 262-269.

8. El-Seedy A, Dudognon T, Bilan F, Pasquet MC, Reboul MP, et al. (2009) Influence of the duplication of CFTR exon 9 and its flanking sequences on diagnosis of cystic fibrosis mutations. J Mol Diagn 11: 488-493.

9. El-Seedy A, Pasquet MC, Bienvenu T, Bieth E, Audrezet MP, et al. (2013) Consequences of partial duplications of the human CFTR gene on $\mathrm{cf}$ diagnosis: mutations or ectopic variations. J Cyst Fibros 12: 407-410.

10. Pagani F, Buratti E, Stuani C, Romano M, Zuccato E, et al. (2000) Splicing factors induce cystic fibrosis transmembrane regulator exon 9 skipping through a nonevolutionary conserved intronic element. J Biol Chem 275: 21041-21047.

11. Farhat R, El-Seedy A, El-Moussaoui K, Paqsquet M-C, Adolphe C, et al. (2015) Multi-physiopathological consequences of the c.1392G>T CFTR mutation revealed by clinical and cellular investigations. Biochem Cell Biol 93: 28-37.

12. Maquat LE, Carmichael GG (2001) Quality control of mRNA function. Cell 104: 173-176.

13. Cheng SH, Gregory RJ, Marshall J, Paul S, Souza DW, et al. (1990) Defective intracellular transport and processing of CFTR is the molecular basis of most cystic fibrosis. Cell 63: 827-834.
14. Fernandez Alanis E, Pinotti M, Dal Mas A, Balestra D, Cavallari N, et al. (2012) An exon-specific U1 small nuclear RNA (snRNA) strategy to correct splicing defects. Hum Mol Genet 21: 2389-2398.

15. Niksic M, Romano M, Buratti E, Pagani F, Baralle FE (1999) Functional analysis of cis-acting elements regulating the alternative splicing of human CFTR exon 9. Hum Mol Genet 8: 2339-2349.

16. Disset A, Michot C, Harris A, Buratti E, Claustres M, et al. (2005) A T3 allele in the CFTR gene exacerbates exon 9 skipping in vas deferens and epididymal cell lines and is associated with Congenital Bilateral Absence of Vas Deferens (CBAVD). Hum Mutat 25: 72-81.

17. Buratti E, Baralle FE (2001) Characterization and functional implications of the RNA binding properties of nuclear factor TDP-43, a novel splicing regulator of CFTR exon 9. J Biol Chem 276: 36337-36343.

18. Welsh MJ, Ramsey BW, Accurso F, Cutting GR (2001) Cystic fibrosis. In: The metabolic and molecular bases of inherited disease (8thedn), McGraw-Hill, New York.

19. Hinzpeter A, Aissat A, Sondo E, Costa C, Arous N, et al. (2010) Alternative splicing at a NAGNAG acceptor site as a novel phenotype modifier. PLoS Genet 6. 